\title{
Convection in a vibrated granular layer
}

\author{
A. Garcimartín ${ }^{\text {a }, ~ J . M . ~ P a s t o r, ~ R . ~ A r e ́ v a l o, ~ a n d ~ D . ~ M a z a ~}$ \\ Departamento de Física y Matemática Aplicada, Facultad de Ciencias, Universidad de Navarra, \\ 31080 Pamplona, Spain
}

\begin{abstract}
When a granular layer is submitted to an oscillating acceleration with a peak value larger than gravity, a large scale motion develops. This movement is in some ways similar to the one displayed by a liquid heated from below, and it is called granular convection. Different conditions beside the parameters of the forcing can affect it, such as the presence of an interstitial gas or the roughness of the walls. We have carried out an experiment to study the convective movement of a granular layer with a temporal resolution high enough to describe the motion of individual grains within one oscillating period. We also present experimental results concerning the friction that the lateral walls exert on the grains and its relevance on granular convection.
\end{abstract}

\section{Introduction}

An isolated system in equilibrium will always evolve so as to increase its entropy or at least to keep it constant. Typically this means that the system is fully disordered and that it lacks any spatial structure. But this does not hold for a dissipative system, where a continuous supply of energy can lead it towards an ordered dynamic state out of equilibrium. Bénard convection is a paradigmatic example of such a behavior: a liquid layer heated from below dissipates the injected energy by adopting an orderly motion: a pattern of convective cells. The features of these cells depend on geometrical parameters, such as the ratio between the liquid depth and the lateral dimension of the container, and on the characteristics of the fluid. Such kind of behavior is therefore expected for granular media, which are inherently dissipative. This is so because although the material from which the grains are made can have a high restitution coefficient - so that it can be considered elastic - the rate of collisions among grains is so large that it usually causes a rapid dissipation of energy. It is therefore not surprising that ordered states out of equilibrium appear easily when energy is supplied to a granular media $[1,2]$.

Injecting energy into such a system to drive it into a dynamical state can be accomplished in four ways: shearing, shaking, through a fluid drag, and under the action of gravity. Here we will deal with the out of equilibrium dynamical states that appear when a granular layer is subjected to a vibration in the same direction than gravity. In 1831, M. Faraday [3] reported that grains deposited on a vibrating plate formed a heap, and investigated the influence of the air on such an structure. The heap has an internal circulation: grains go up under de apex, descend down the sides and go towards the center at the base, closing the trajectory. Faraday's work was pioneering in the sense that it is one of the first reports concerning the self-organization of an out of equilibrium system. Nevertheless, not much attention was paid to this phenomenon until a couple of decades ago. In 1988, S.B. Savage [4] described the motion of beads in a rectangular box with a vibrating flexible base. The fixed side walls and the fact that the vibration at the base was spatially non-uniform differs from most experiments carried out since then, but many results remain qualitatively unchanged. He observed symmetric

\footnotetext{
a e-mail: angel@fisica.unav.es
} 
closed vortices in which the velocity was upwards at the vertical centreline and downwards along the vertical sidewalls, resembling the convective cells in heated liquid layers. Maybe due to this likeness this motion is now known as granular convection. Shortly after this work, Evesque and Rajchenbach [5] demonstrated experimentally that the pertinent control parameter is the peak acceleration of the container. (Usually, the excitation is a harmonic oscillation, and the peak acceleration is normalized to gravity to produce an adimensional quantity: $\Gamma=\frac{A f^{2}}{4 \pi^{2} g}$, where $A$ is the amplitude and $f$ is the frequency of the driving).

Despite the apparent simplicity of this phenomenon, the origin of the coherent movements observed in the experiments is not well understood. In Bénard-Marangoni convection, the typical honeycomb pattern appears when the influence of the lateral boundaries can be neglected [6], and in the case of small aspect ratio systems boundary conditions completely define the resulting pattern [7]. Unfortunately, a simple analogy with the granular convection can not be introduced. For instance, the convective threshold in a heated liquid layer is well defined through the dimensionless Rayleigh number. This number depends on the geometrical parameters - such as the liquid depth - and on the departure from the equilibrium state, which is quantified by the temperature difference between the top and the bottom surfaces. The instability threshold can be expressed as the ratio between the time scales of the energy input and the dissipative processes. In granular media, on the other hand, it is difficult to identify such temporal and geometrical scales. Moreover, the depth of the material can determine the kind of instability that will appear. For a shallow granular layer (it is generally understood that this means that the depth is smaller than about ten grain diameters) there is not a large scale circulation: instead, waves of different kinds appear [8]. If, on the other hand, the height of the confined layer is large enough, collective large scale circulation (spanning over the whole layer) can be observed.

Laroche [9] and Clément [10] proceeded to describe convection and raised for the first time the question of the influence of the lateral walls and the presence of interstitial air in this circulation. Another subject raised in those papers was the "fluidization" of the granular medium, meaning that the vibrated grains flow like a liquid. Further work uncovered the relevance of three possible mechanisms for the granular convection. Pak and collaborators [11] studied the influence of the interstitial gas and found that convection is greatly impaired in an evacuated contained. Arguments still go on about the significance of this finding, although many believe that air acts mainly as a lubricant. Convection therefore would stop in the absence of air because of internal friction among grains, but air - if this is true - would not directly cause convection. Knight et al. [12] reported measurements of the velocity of a tracer particle inside a tapped granular layer, obtained by magnetic resonance imaging. They concluded that the friction with walls was related to the streaming velocity. Finally, numerical simulations [13] showed that a thermal diffusion from the vibrating plate could cause similar motions in diluted granular gases. For a dilute gas (where dissipation is not so fast as in a dense granular medium) hydrodynamic theories can be used to describe the problem by introducing a temperature obtained from the mean kinetic energy. The diffusion of the kinetic energy can drive convection, but this mechanism seems to be restricted to fluidized grains with low densities.

In this article, we will first describe our experiment, and provide an insight into the measurements that are accessible with our equipment. We will first present results concerning the motion of the whole granular layer, which shows a period doubling bifurcation in the flight time as the control parameter is increased. In this section, the influence of interstitial air will be analyzed. We will then turn to the motion of individual grains near the walls in order to study the cause for the relative motion within the granular layer (the granular convection) and describe the patterns that appear. In the last section, we will offer some conclusions.

\section{Experimental setup}

In order to describe the motion of the granular layer, we will rely mainly on two measurements: the instantaneous acceleration of the excitation and the velocity of the grains. The first one can be easily obtained by attaching an accelerometer to the container. Its signal is picked up 


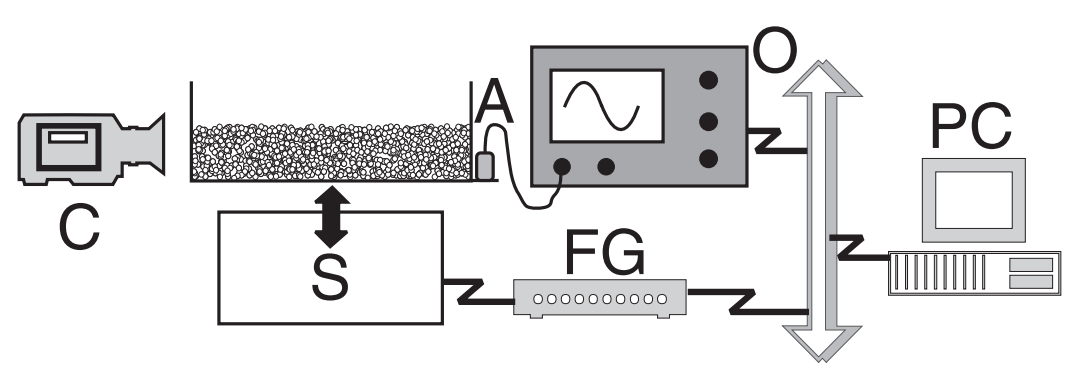

Fig. 1. Sketch of the experimental setup. C: High-speed camera; S: shaker; FG: function generator; A: accelerometer; O: oscilloscope; PC: computer.

by an oscilloscope and data are registered by a computer (see figure 1). The velocity of the grains has been obtained by particle tracking. A high-speed camera (Photron FASTCAM X$1024 \mathrm{PCI}$ ) is used to record a movie at a suitable frame rate, depending on the case. Typically, 1000 frames per second is enough to resolve the "fast" motion of the grains within one vibrating cycle. The movie is then downloaded to a computer, where individual grains are tracked with an image processing software. Standard particle tracking techniques provide a resolution much better than the particle diameter. Spatio-temporal diagrams can also be used to obtain the velocities of the grains. Additional information about this technique can be found in [14].

The container is attached to the shaft of an electromechanical shaker (TiraVib 52100) capable of delivering a sinusoidal acceleration with a peak value up to $10 \mathrm{~g}$. The shaker is feeded from a function generator (Stanford Research DS 345) which is in turn commanded from a computer. In this way, the experiment can be automated and the parameter $\Gamma$ can be easily controlled. In this experiment the frequency is fixed at $f=110 \mathrm{~Hz}$ and $\Gamma$ is varied by changing the vibration amplitude.

Almost any granular material can be used to observe convection. As a large number of beads is desirable, the grains must be small. On the other hand, when grains are too small, cohesive forces due to humidity and electrostatic forces can exceed the weight of a grain, and undesirable effects appear. Glass beads with a diameter $d=0.43 \pm 0.1 \mathrm{~mm}$ offer a good compromise and have been used in this experiment. The density of the glass is $\rho_{b}=2.5 \mathrm{~g} / \mathrm{cm}^{3}$.

Different containers have been used to study the effect of the lateral walls on granular convection. They will be described later with the corresponding results. All of them are cylindrical boxes with an inner diameter of $52 \mathrm{~mm}$, so that the diameter of the container is much larger than the diameter of one bead. The height of the layer is measured in units of particle diameters, i.e., $N=h / d$, where $h$ is the height of the layer. Granular convection is usually observed if $N \geq 10$, a threshold for which an explanation has been offered [2]. In our experiment $N$ is always greater than 10 .

\section{Bifurcations}

The dynamics of the granular layer as a whole is determined by the amount of energy injected, which is quantified by the control parameter $\Gamma$, as suggested in [5]. As the frequency is fixed at $f=110 \mathrm{~Hz}$ (the choice of this particular value is not crucial), $\Gamma$ is varied by changing the amplitude of the oscillations. As the control parameter is increased, the sequence of events for the whole layer is the following. For $\Gamma<1$ the granular layer will remain in contact with the container during all the cycle. But when the peak acceleration of the forcing overcomes the gravity, the layer will loose contact with the vibrating plate and perform a "free" flight. Therefore the primary instability is expected to take place at $\Gamma=1$, because the grains will then be allowed to move while performing their flight.

Just above the threshold, the motion of a the granular layer consists of cyclic jumps at the same frequency than the forcing. The layer begins to fly at the moment in the cycle where the instantaneous value of the acceleration is equal to $g$. The value of the velocity and the phase at this precise instant provide the initial conditions for the flight. Gravity and whatever forces 
act on the granular layer cause a downward acceleration. The layer falls and it lands when it bumps into the base. As it is composed of a large number of particles, it can be deemed as an almost perfectly inelastic body, so the energy is completely lost at the collision. Therefore the layer gets stuck to the vibrating plate. It begins to fly again when the instantaneous acceleration overcomes the gravity. Let us call $T$ the period of the forcing (as $f$ is fixed at $110 \mathrm{~Hz}$, $T=0.00909 \mathrm{~s}$ ); then the granular layer spends a time $\tau$ flying and a time $T-\tau$ stuck to the base. The flight time $\tau$ can be obtained from the accelerometer signal (see for example [8], figure 3), because the impact of the granular layer against the vibrating plate leaves an imprint on the acceleration.

As $\Gamma$ is increased, so does the flight time. But if flight times are calculated by assuming that the granular layer is a perfectly inelastic body upon which only gravity is acting, then the experimental results are not recovered. The measured flight times are shorter than predicted [15]. Kroll [16] pointed out that the effect of interstitial air was likely to be the cause. Gutman [17] proposed an improved model that included the compressibility of air. In brief, the following simplifying assumptions are made [18]: (1) The granular layer can be likened to a porous piston (2) The collision between the piston and the vibrating plate is almost instantaneous when compared to the vibrating period (3) Friction against walls is negligible (4) Permeation of air through the grains can be described by Darcy's law (5) The porosity of the granular layer is constant.

From these hypothesis, the following can be deduced [18]. Darcy's law states that pressure drop in the granular layer must be proportional to the velocity $u$ with which air passes through it, so

$$
\frac{\partial P}{\partial z}=\frac{\mu}{\kappa} u
$$

Here $P$ is the pressure, $z$ is the vertical coordinate, $\mu$ is the air viscosity and $\kappa$ is the porosity of the granular layer, that can be approximated by the Carman-Kozeny expression [19]

$$
\kappa=\frac{\varepsilon^{3}}{180(1-\varepsilon)^{2}} d^{2}
$$

In this equation $\phi=1-\varepsilon$ is the compaction fraction. Under Kroll's assumption (5) it can be shown that the pressure $P$ inside the granular medium obeys the following diffusion equation $[20]$

$$
\frac{\partial P}{\partial t}=\frac{P_{0} \kappa}{\mu(1-\varepsilon) \frac{\partial^{2} P}{\partial^{2} z}}
$$

where $P_{0}$ is the atmospheric pressure. In order to solve equation (3) the following boundary conditions are used. At the top of the layer, $P_{z=0}=P_{0}$. And at the bottom of the layer $(z=h)$, the continuity equation holds that

$$
\frac{\mathrm{d} \rho_{g}}{\mathrm{~d} t}=\rho_{g, z=0}, \quad u=-\rho_{g, z=0} \frac{\kappa}{\mu} \frac{\partial P}{\partial z}
$$

where $\rho_{g, z=0}$ is the gas density at $P_{0}$ and $s$ is the height of the air gap between the vibrating plate and the granular layer. If the pressure change is small compared to $P_{0}$, then $\rho_{g}$ does never differ a lot from $\rho_{g, z=0}$, so $s\left(d \rho_{g} / d t\right) \ll \rho_{g, z=0}(\mathrm{~d} s / \mathrm{d} t)$ and equation 4 now reads

$$
\frac{\mathrm{d} s}{\mathrm{~d} t}=-\left.\frac{\kappa}{\mu} \frac{\partial P}{\partial z}\right|_{z=h}
$$

In the laboratory reference frame, the equation of motion for the granular layer is

$$
\frac{\mathrm{d}^{2}(s+x)}{\mathrm{d} t^{2}}=-g+\frac{P_{z=h}-P_{0}}{\rho_{b} \varepsilon h}
$$

where $x(t)$ is the driving vibration and $\rho_{b}$ is the density of the beads. By integrating equations (3)-(6) in one dimension, it is possible to obtain the pressure difference in a cycle, as well as the trajectory of the mass center of the granular layer and its flight time. 


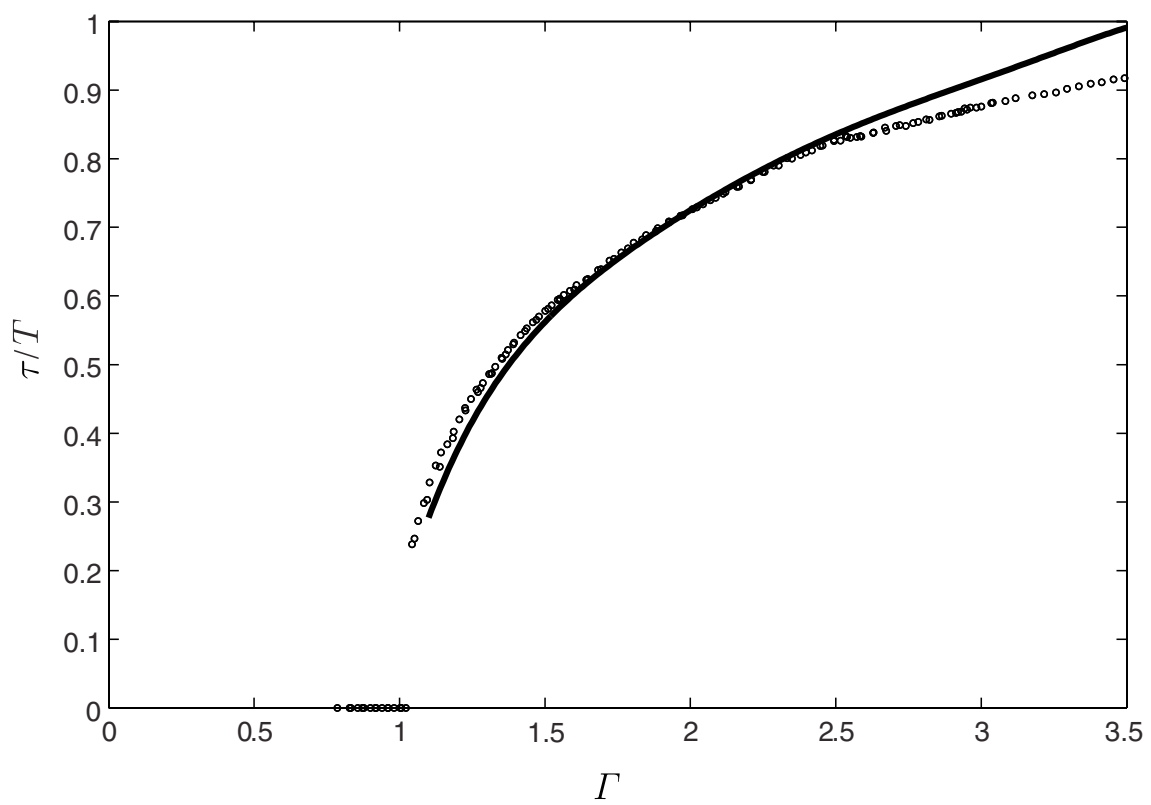

Fig. 2. Flight times $\tau$ normalized to the driving period $T$ as a function of $\Gamma$. Results obtained from the Gutman model (solid line) are shown along with experimental data (open circles). The results where obtained for $N=20, \phi=1-\varepsilon=0.59$ and $\mu=1.8 \times 10^{-5} \mathrm{~kg} \mathrm{~m}^{-2} \mathrm{~s}^{-1}$.

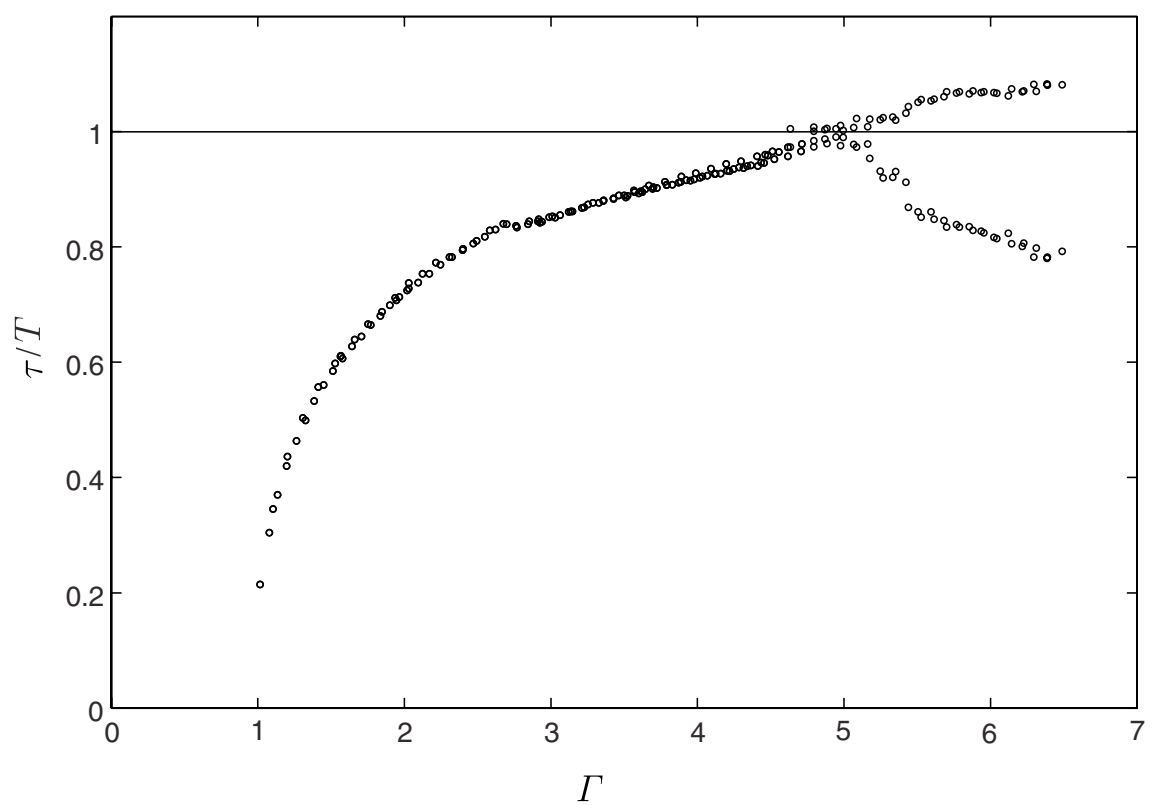

Fig. 3. The flight time for a granular layer of $N=33$ as a function of $\Gamma$. Flight times $\tau$ are normalized to the driving period $T$. The horizontal solid line marks $\tau=T$.

The calculated flight times are represented in figure 2 along with the experimental measurements. It is clear that the Gutman model correctly reproduces the flight times of the granular layer as a whole up to $\Gamma \approx 2.3$. The disagreement between the model and the experiment for greater driving accelerations is not due to the presence of air; the finite duration of the collision (assumption (2)) is likely to be involved [15]. 


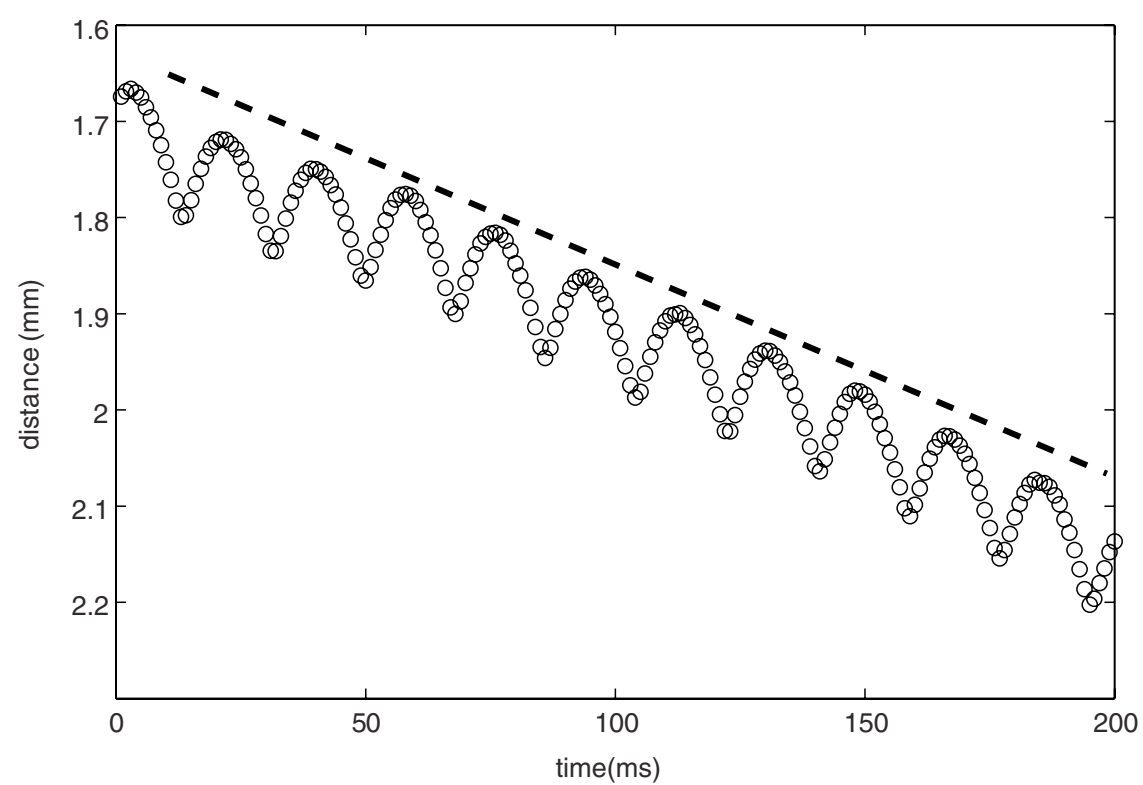

Fig. 4. Position of a single bead tracked near the lateral wall $(\Gamma=2.6$ and $N=100)$. The dashed line is the slow drift that makes up the convective motion (the line itself is not a fit but just a guide for the eye). Vertical distances are positive downward; their origin is arbitrary.

If $\Gamma$ is increased further, the flight time $\tau$ reaches the value of the driving period $T$. A period doubling bifurcation takes place, because the granular layer performs long flights (longer than $T$ ) alternated with short flights (shorter than $T$ ). This is displayed in figure 3 . Thus, every $2 T$, the grains in the layer will perform one short flight and one long flight (apart from a certain time that they will spent stuck to the vibrating plate).

\section{Patterns}

The dynamics of the whole granular layer, as described in the previous section, can be understood - at least for moderate values of the acceleration - in terms of a porous piston which collides inelastically with the vibrating plate at each cycle. Nevertheless, the movement of the granular layer as a whole does not provide the complete picture. In fact, inside the layer the grains move differently from one place to another, and this differential motion establishes the convective patterns. Near the onset (for $\Gamma \gtrsim 1$ ) the velocity field takes the form of a toroidal roll, as depicted in [15], figure 5. This differential motion can only be understood if there are forces acting only at certain zones of the granular layer, affecting exclusively some of the grains. It is therefore reasonable to look at the grains near the wall, because lateral walls and their possible effects are not taken into account in the model used in the previous section, and any effect they may produce will be local, so it might cause the granular convection.

In order to investigate the influence of the walls, we have tracked single grains near the lateral wall. One of the obtained trajectories is depicted in figure 4. At each cycle, the individual grain performs a flight. It is quite easy to spot the landings, because the velocity changes abruptly, but the take off cannot be pinpointed. The initial velocity is determined by the instantaneous speed of the plate at the moment of the take off, and the downwards acceleration must be caused by gravity and whatever other forces (friction, etc.) are acting on it. These periodic "jumps" have the same frequency than the forcing, at least near the onset (where $\Gamma \gtrsim 1$ ). This is what can be called the "fast" motion.

But there is a conspicuous feature of the motion of a bead near the lateral wall: it descends along the wall. As can be noticed in figure 4, each landing position is lower than the previous 


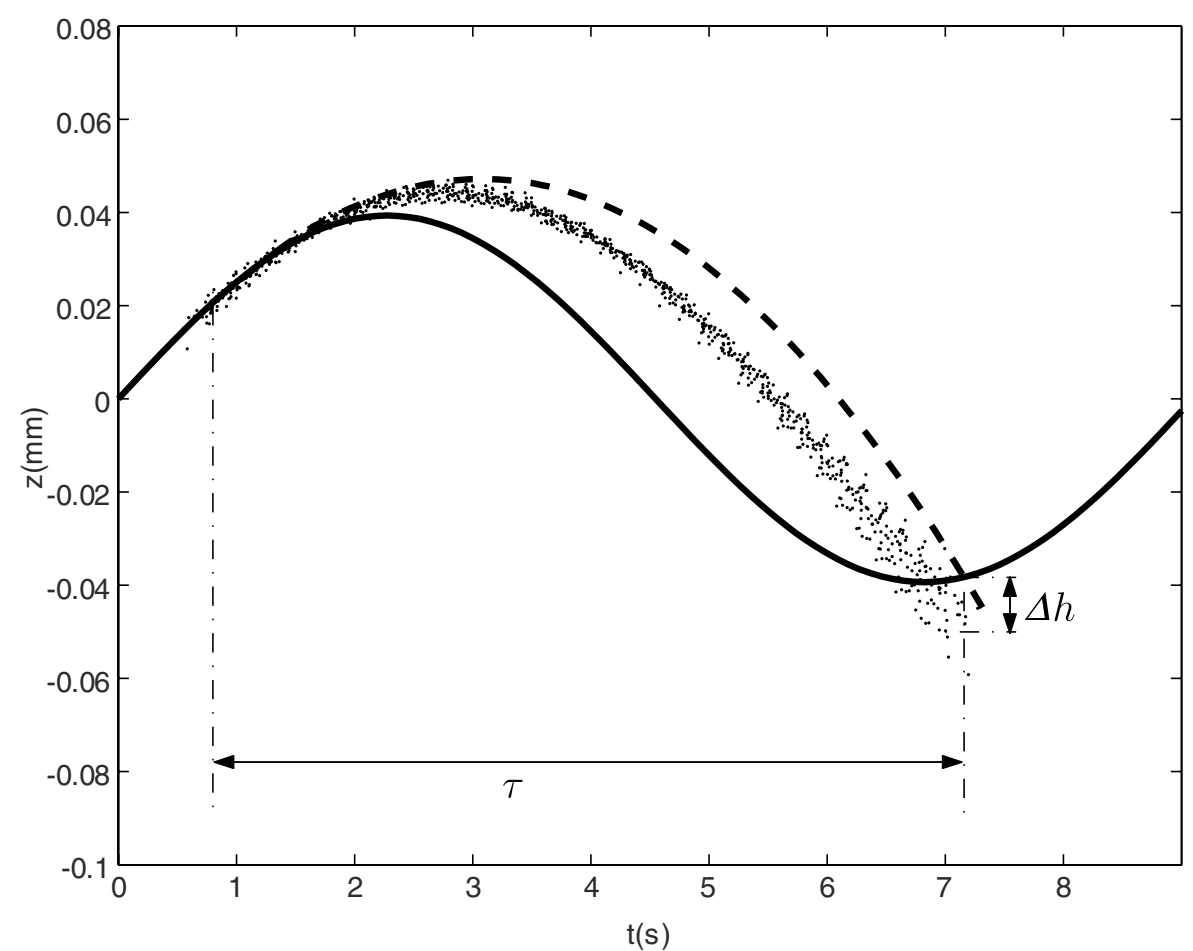

Fig. 5. The positions of the grains near the lateral wall, as obtained from particle tracking (dots). All the data have been repositioned to an arbitrary initial position, keeping the phase information. The solid line is the position of the vibrating plate. The results where obtained for $\Gamma=1.9$ and $N=20$. Also shown is the trajectory for mass center as obtained from the Gutman model (dashed line).

one. This means that another force, apart from gravity, must be acting upon the bead so as to produce a net drift downwards. This drift is shown as a dashed line in the plot. We can therefore distinguish between a fast motion, which are the jumps at the forcing frequency, and a slow motion, that is the downward drift. This slow downward drift of the grains, that takes place near the walls, is at the origin of the convective motion. Grains descend along the lateral wall, travel radially towards the axis at the bottom plate, rise near the center of the layer and move outwards at the upper surface, closing the flow.

In order to quantify the effect of the lateral wall, the motion of individual grains adjacent to the container wall is to be compared to the motion of the granular layer as a whole. This shown in figure 5 for $\Gamma=1.9$, where the positions of large number of grains, taken to a common reference, are plotted with small dots. Along with it, the trajectory of the mass center is displayed as obtained from the porous piston model, which describes it quite correctly for $\Gamma=1.9$, as can be seen in figure 2 .

The most obvious peculiarity of these measurements is the difference between the trajectories of the grains near the wall and the motion of the mass center of the granular layer. This relative motion causes the convection. In fact, if we take the difference between the landing point of the mass center and the landing point of the grains near the wall ( $\Delta h$ in figure 5$)$ and divide it by $T$, we get roughly the convection velocity (albeit with a large uncertainty; we never used this method to obtain the velocities). This means that another force, apart from gravity and the effect due to the interstitial air, is acting on the grains near the wall so as to produce a net downward motion at each cycle. The quantification of this force by means of an effective acceleration will be presented elsewhere [15].

We have also changed the properties of the lateral wall in order to investigate their influence on the convection. This was accomplished, for example, by gluing sandpaper (with a grain of 150 microns and a grain of 30 microns) to the wall, or by using a cell made of steel into which 
Table 1. Reference velocities for different wall materials, for $\Gamma=2$.

\begin{tabular}{ll}
\hline Material & Velocity $(\mathrm{mm} / \mathrm{s})$ \\
\hline glass & 1.2 \\
smooth steel & 1.1 \\
grooved steel & 0.4 \\
sandpaper, 30 microns & 1.2 \\
sandpaper, 100 microns & 0.4 \\
\hline
\end{tabular}

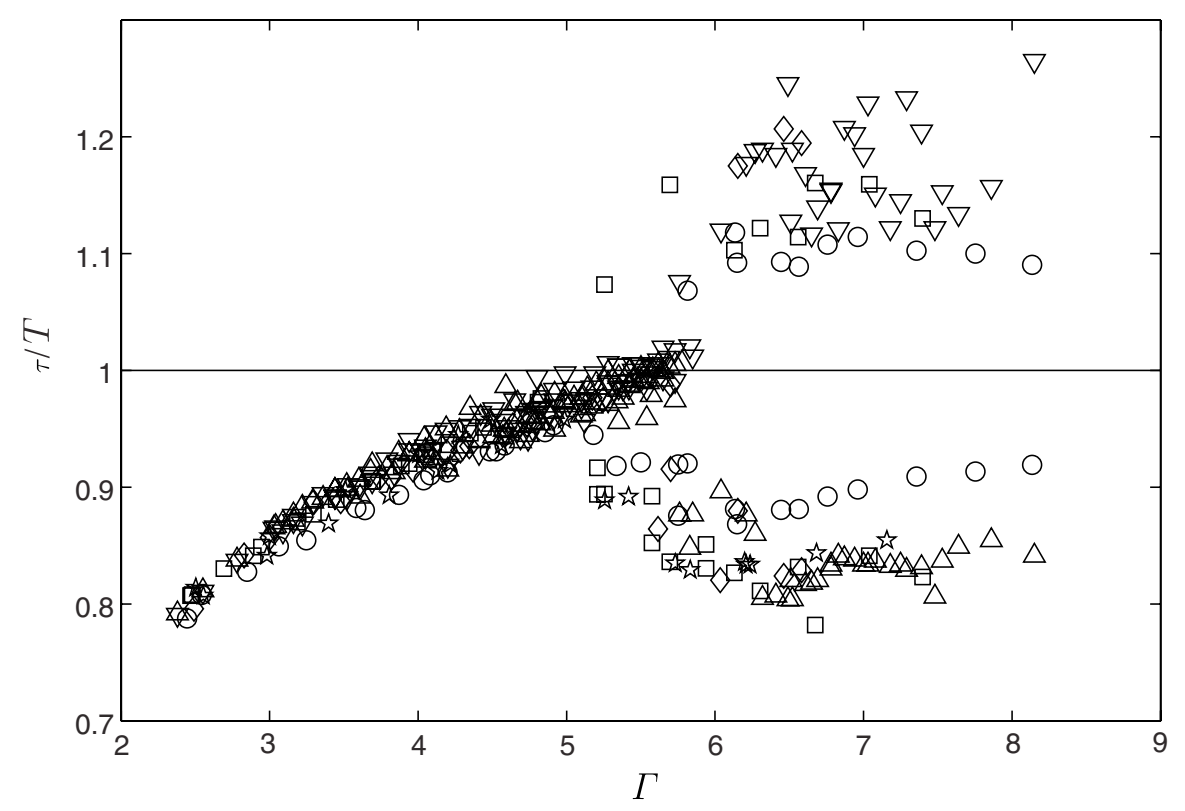

Fig. 6. Flight times for the granular layer as a whole near the bifurcation point for lateral walls having different properties (each symbol corresponds to one of them). The flight times are normalized to the forcing period $T$.

grooves were carved. For each one we measured the velocity of the beads at the same point on the upper surface. This value is meaningful if we use it just to compare the different cases. The values obtained are shown in table 1 . The value reported is the mean velocity (i.e. averaged over many cycles) at a point on the upper surface of the granular layer $1 \mathrm{~cm}$ apart from the lateral wall (therefore these velocities have a radial direction).

From these results it stems that smooth walls are associated with higher convection velocities. It is probable that in changing the lateral wall we are mainly modifying the static friction coefficient, and the effect of this would be to reduce the flight time of the grains near the wall, which in turn would switch off the driving for their relative movement by keeping the grains stuck to the wall. Note that if grains take off at a later phase then the initial velocity for the flight would be smaller, and the flight shorter. In this regard, taps and a sinusoidal vibration may well reveal to produce different results [12], although this aspect should be studied in more depth.

It is important to note that the velocity profiles have the same shape (the profile along the vertical wall is shown in [14] for glass), and they only change by a scale factor. Moreover, the flight times measured from the accelerometer signal (corresponding to the mass center) is always the same before the bifurcation point (see figure 6). This means that the wall properties do not affect the flight time and the dynamics of the granular layer as a whole, but they do affect the convection velocity. 

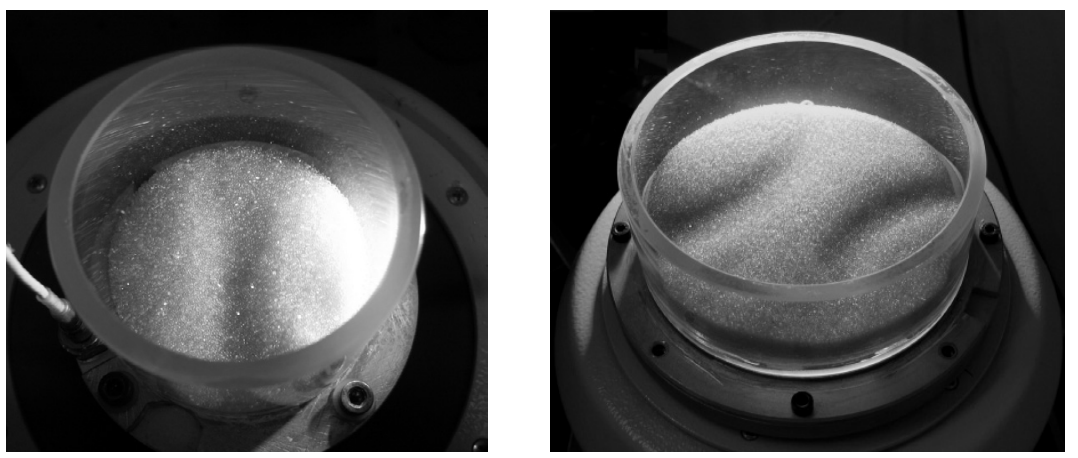

Fig. 7. The vibrated granular layer under lateral lighting shows different regions (two in the one at the left, three in the one at the right) that are performing short and long flights out of step with respect to the adjacent one. The regions are visible because a small heap is formed in each of them. Note that the frontiers between them finish perpendicularly to the wall.

After the bifurcation point at $\tau=T$ new patterns can appear. The grains must perform one short flight and one long flight in a time span of $2 T$, but the granular layer can do this in two ways: a short flight followed by a long flight, or a long flight followed by a short flight. The possibility that one zone of the granular layer performs one kind of flight while other zone is performing the other is at the origin of the patterns sometimes seen in convecting granular layers above the value of $\Gamma$ corresponding to the period doubling bifurcation for the flight times. Two examples of such patterns are shown in figure 7 . The photographs show the upper surface of the granular layer under lateral lighting. Distinct regions can be seen because the surface is not flat: small heaps form over each zone. An interesting feature that remains unexplained is that the borders between these regions always try to be perpendicular to the lateral wall, like in thermal convection (see also [14], figure 2).

\section{Conclusions}

The experiments reported here allowed us to investigate the factors that influence the motion of a vibrated granular layer. For the granular layer as a whole, a free flight under the action of gravity alone that ends with an inelastic collision will not reproduce the experimental results. Nevertheless, taking into account the effect of interstitial air the experimental results are faithfully reproduced for moderate values of the dimensionless acceleration $(\Gamma \lesssim 2.3)$. For $\Gamma$ beyond that value, the model fails to reproduce the experimental results. The disagreement is not caused by the presence of air, and more work is needed to understand the phenomenon.

It has been shown that near the lateral wall the grains suffer a downward drift which is caused by a local force acting on them. This drift is at the origin of granular convection. By changing the properties of the wall we have shown that the dynamics of the layer as a whole is not changed (the flight times are the same and the bifurcation point does not change significantly), but the convection velocity is greatly influenced. This means that the extra force responsible for the differential movement that the grains carry out is a local effect of the walls.

This work has been funded by Spanish Government project FIS 2005-03881 and by the Universidad de Navarra (PIUNA). J.M.P. and R.A. thank the Asociación de Amigos de la Universidad de Navarra for a fellowship. We are also grateful to I. Zuriguel for his comments and suggestions.

We are indebted to Carlos Pérez García in many ways; we would like just to mention his support when some of us started the line of research concerning granular media in our laboratory. 


\section{References}

1. G.H. Ristow, Pattern Formation in Granular Materials (Springer, Berlin, 2000)

2. J. Duran, Sables, poudres et grains (Eyrolles, Paris, 1997)

3. M. Faraday, Philos. Trans. R. Soc. Lond. 52, 299 (1831)

4. S.B. Savage, J. Fluid Mech. 194, 457 (1988)

5. P. Evesque, J. Rajchenbach, Phys. Rev. Lett. 62, 44 (1989)

6. E.L. Koschmieder, Bénard Cells and Taylor Vortices (Cambridge University Press, Cambridge, 1993)

7. T. Ondarçuhu, J. Millán-Rodríguez, H.L. Mancini, A. Garcimartín, C. Pérez-García, Phys. Rev. E 48, 1051 (1993)

8. F. Melo, P.B. Umbanhowar, H.L. Swinney, Phys. Rev. Lett. 75, 3838 (1995)

9. C. Laroche, S. Douady, S. Fauve, J. Phys. (France) 50, 699 (1989)

10. E. Clément, J. Duran, J. Rajchenbach, Phys. Rev. Lett. 69, 1189 (1992)

11. H.K. Pak, E. Van Doorn, R.P. Behringer, Phys. Rev. Lett. 74, 4643 (1995)

12. J.B. Knight, E.E. Ehrichs, V.Y. Kuperman, J.K. Flint, H.M. Jaeger, S.R. Nagel, Phys. Rev. E 54, 5726 (1996)

13. R. Ramírez, D. Risso, P. Cordero, Phys. Rev. Lett. 85, 1230 (2000)

14. A. Garcimartín, D. Maza, J.L. Ilquimiche, I. Zuriguel, Phys. Rev. E 65, 031303-1-5 (2002)

15. J.M. Pastor, D. Maza, I. Zuriguel, J.-F. Boudet, A. Garcimartín, Physica D (submitted)

16. W. Kroll, Forschung auf der Gebiete des Ingenieurwesen 20, 2 (1954)

17. R.G. Gutman, Trans. Instn. Chem. Engrs. 54, 174 (1976)

18. M.E. Möbius, X. Cheng, P. Eshuis, G.S. Karczmar, S.R. Nagel, H.M. Jaeger, Phys. Rev. E 72, 011304-1-13 (2005)

19. R.M. Nedderman, Statics and Kinematics of Granular Materials (Cambridge University Press, Cambridge, 1992)

20. P.C. Carman, Flow of Gases Through Porous Media (Butterworth Scientific, London, 1956) 